\title{
Heavy-Ion-Induced Soft Breakdown of Thin Gate Oxides
}

\author{
J. F. Conley, Jr., J. S. Suehle, A. H. Johnston, B. Wang, T. Miyahara, E. M. Vogel, and J. B. Bernstein
}

\begin{abstract}
Heavy-ion-induced soft and hard breakdown are investigated in thin gate oxides as a function of linear energy transfer, fluence, and voltage applied during irradiation. It is found that postirradiation oxide conduction is well described by the Suñé quantum point contact model.
\end{abstract}

Index Terms-Dielectric breakdown, gate oxide, heavy ions, microelectronics, MOS, radiation, $\mathrm{SiO}_{2}$.

\section{INTRODUCTION}

$\mathbf{F}$ UTURE Jet Propulsion Laboratory missions to the outer planets will require electronics with ultrahigh levels of radiation tolerance $(>1 \mathrm{Mrad})$. Although radiation produces only a modest shift in the threshold voltage of the thin gate oxides used in advanced MOS technology, these thin oxides were still susceptible to radiation effects. In the last few years, pioneering work has uncovered a variety of new radiation-induced effects in thin gate oxides [1]-[10], including radiation-induced leakage current (RILC) in gamma and electron irradiated oxides [1]-[5] and single-event gate rupture (SEGR) [6]-[8] and radiation-induced soft breakdown (RSB) [7]-[10] in oxides exposed to high linear energy transfer (LET) heavy-ion irradiation. Despite this work, the effects of radiation on the reliability of ultrathin oxides $(<4 \mathrm{~nm})$ have not been extensively characterized. In this paper, we report on heavy-ion-induced soft breakdown of 3.0- and 3.2-nm thin oxide films as a function of LET, fluence, and the voltage applied during irradiation. Postirradiation oxide conduction is modeled with the Suñé quantum point contact model [11].

\section{EXPERIMENTAL DETAILS}

Two sets of oxide test capacitors were used in this study. P-well test capacitors supplied by a commercial facility had an oxide thickness of $3.2 \mathrm{~nm}$, a poly-Si gate thickness of approximately $400 \mathrm{~nm}$, and an area of $10^{-3} \mathrm{~cm}^{2}$. P-substrate capacitors supplied by a university had an oxide thickness of $3.0 \mathrm{~nm}$, a poly thickness of $400 \mathrm{~nm}$, and areas of $10^{-4} \mathrm{~cm}^{2}$ and $4 \times$ $10^{-4} \mathrm{~cm}^{2}$. Oxide thickness was determined by high-frequency capacitance versus voltage curves using simulation to account for substrate quantum confinement and poly depletion effects.

Manuscript received July 17, 2001. This work was supported under contract by the National Aeronautics and Space Administration.

J. F. Conley, Jr., A. H. Johnston, and T. Miyahira are with the NASA Jet Propulsion Laboratory, California Institute of Technology, Pasadena, CA 91109 USA (e-mail: john.f.conley@jpl.nasa.gov).

J. S. Suehle and E. M. Vogel are with the Semiconductor Electronics Division, National Institute of Standards and Technology, Gaithersburg, MD 20899 USA

B. Wang and J. B. Bernstein are with the Center for Reliability Engineering, University of Maryland, College Park, MD 20742 USA.

Publisher Item Identifier S 0018-9499(01)10669-6.

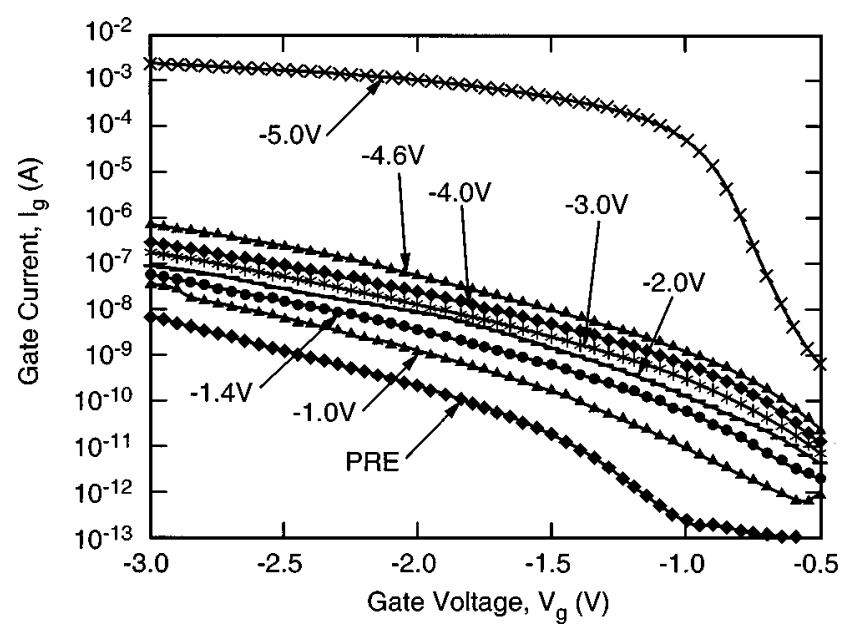

Fig. 1. $I_{g}$ versus $V_{g}$ curves as a function of $V_{\text {app }}$ for a 3.2-nm $10^{-3} \mathrm{~cm}^{2}$ capacitor exposed to Au $(\mathrm{LET}=80)$.

The intrinsic breakdown strength of both the 3.2- and 3.0-nm oxides was $\geq 17 \mathrm{MV} / \mathrm{cm}$, determined by a standard voltage ramp test. The reliability and quality of the commercial and university oxides are approximately the same. The charge to breakdown $\left(Q_{\mathrm{bd}}\right)$ as a function of gate voltage overlapped for the two sets of oxides.

Heavy-ion testing was conducted at the Brookhaven National Laboratories Tandem Van de Graff accelerator using $343 \mathrm{MeV}{ }^{197} \mathrm{Au}, 343 \mathrm{MeV}{ }^{127} \mathrm{I}$, and $279 \mathrm{MeV}^{81} \mathrm{Br}$ (respective LETs $=81.9,59.9$, and $37.5 \mathrm{MeV}-\mathrm{cm}^{2} / \mathrm{mg}$ ) and at the Texas A\&M University Cyclotron using $2955 \mathrm{MeV}{ }^{197} \mathrm{Au}, 737 \mathrm{MeV}$ ${ }^{129} \mathrm{Xe}$, and $2861 \mathrm{MeV}{ }^{129} \mathrm{Xe}$ (respective LETs $\cong 80,60$, and $40 \mathrm{MeV}-\mathrm{cm}^{2} / \mathrm{mg}$ ). Exposures were conducted at normal incidence with capacitor gates either tied to ground or biased in accumulation.

A series of irradiations was performed sequentially on each sample. For each irradiation step $V_{\text {app }}$, the gate voltage applied during irradiation was held constant while the capacitor was exposed to a set fluence of ions. Multiple devices were irradiated at a time. $I_{g}-V_{g}$ measurements were taken in situ immediately following each irradiation step. Initially, additional $I_{g}-V_{g}$ curves were taken over the next several minutes in order to avoid a transient decrease in the postirradiation excess leakage [9]. After several irradiation steps, the transient effect appeared to dissipate, and thereafter, only one trace per step was taken.

\section{RESULTS}

Fig. 1 shows a typical example of $I_{g}-V_{g}$ curves as a function of $V_{\text {app }}$. Note that oxides were biased in accumulation and $I-V$ curves were taken as described in Section II. In Fig. 1, 


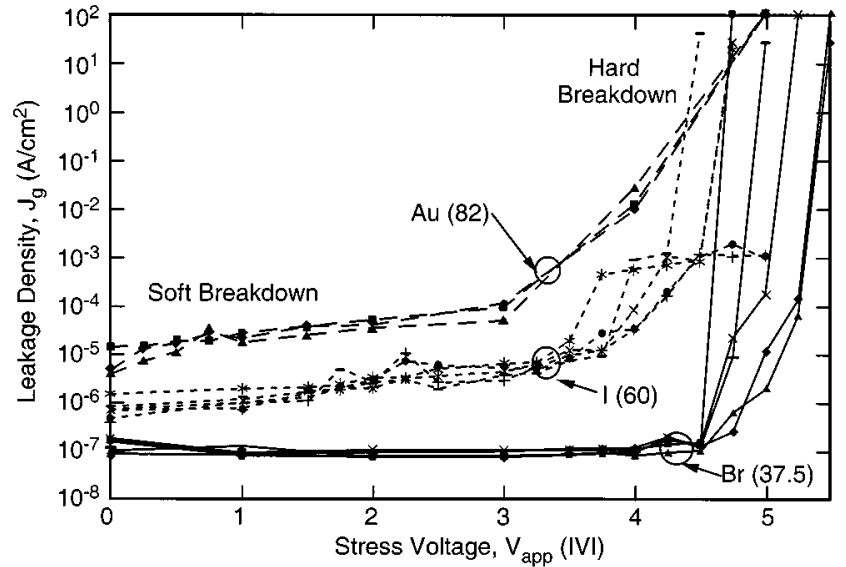

Fig. 2. Plot of $J_{g}$ (extracted at $V_{g}=3.0 \mathrm{~V}$ ) versus $V_{\text {app }}$ for $3.0 \mathrm{~nm}, 10^{-4}$, and $4 \times 10^{-4} \mathrm{~cm}^{2}$ capacitors exposed to Au $($ LET $=82)$, I $($ LET $=60)$, and $\mathrm{Br}(\mathrm{LET}=37.5)$

3.2-nm-thick $10^{-3} \mathrm{~cm}^{2}$ capacitors were exposed to ${ }^{197} \mathrm{Au}$ $($ LET $=80)$ with a fluence of approximately $10^{6} \mathrm{ions} / \mathrm{cm}^{2} / \mathrm{step}$ ( 1000 "hits"/capacitor/step), at a typical flux of roughly 1.9 $\times 10^{4} / \mathrm{cm}^{2} / \mathrm{s}( \pm 15 \%)$.

Fig. 2 shows oxide leakage current density $J_{g}\left(\mathrm{~A} / \mathrm{cm}^{2}\right)$ as a function of $V_{\text {app }}$ for 3.0-nm capacitors exposed to $\mathrm{Au}$, I, or $\mathrm{Br}$, LET $\cong 82,60$, and $37.5 \mathrm{MeV}-\mathrm{cm}^{2} / \mathrm{mg}$, respectively. (Note that different $V_{\text {app }}$ steps were used for each LET.) Oxides were irradiated for $35-140 \mathrm{~s}$ at a typical flux of roughly $7 \times 10^{4} / \mathrm{cm}^{2} / \mathrm{s}$ $( \pm 50 \%)$ to a fluence of approximately $10^{6} \mathrm{ions} / \mathrm{cm}^{2} / \mathrm{step}$. The intrinsic lifetime of the devices for an applied voltage of $-4.9 \mathrm{~V}$ was determined to be approximately $8000 \mathrm{~s}$. Devices at each LET were irradiated together. $J_{g}$ has been extracted at $V_{g}=$ $-3.0 \mathrm{~V}$ in order to quantify the amount of leakage or degree of soft breakdown while assuring that no additional device degradation was induced. The device lifetime at $-3.0 \mathrm{~V}$ was estimated to be $3 \times 10^{7} \mathrm{~s}$. The prestress leakage current density in all cases was approximately $10^{-7} \mathrm{~A} / \mathrm{cm}^{2}$. Results from capacitors with areas of $10^{-4}$ and $4 \times 10^{-4} \mathrm{~cm}^{2}$ are plotted together. The capacitors received approximately 500 and 2000 "hits" per irradiation step, respectively. It is found that radiation-induced leakage is proportional to capacitor area and that leakage current density is the same for both areas, indicating that the excess current is not due to a single leakage path [10].

From Figs. 1 and 2, it is clear that the postradiation oxide conduction in these thin oxides is a function of both LET and bias (as reported in Ceschia et al. [9], [10] and in thicker oxides by Sexton et al. [6], [8] and Johnston et al. [7]). For both $\mathrm{Au}$ and I, the oxides exhibit soft breakdown (SBD) at lower voltages (including $0 \mathrm{~V}$ ) [8]-[10] and hard breakdown (gate rupture) at voltages greater than $3.5 \mathrm{~V}$. The SBD is indicated by the $>10 \times$ increase in $I_{g}$, increased noise, and the exponential dependence of leakage on $\mathrm{V}_{\mathrm{g}}$ (at the higher voltages, $I_{g}-V_{g}$ curves appear as nearly straight lines on the log-linear scale). Note also that subsequent traces are roughly parallel. In the reliability literature, the first SBD is considered to be the end of oxide life [12], [13]. Hard breakdown (HBD) is indicated by an increase in leakage current by over two orders of magnitude and the linear appearance of the $I_{g}-V_{g}$ characteristics on a linear-linear scale (resembling a resistive conductance). For the case of $\mathrm{Br}$, the ox-

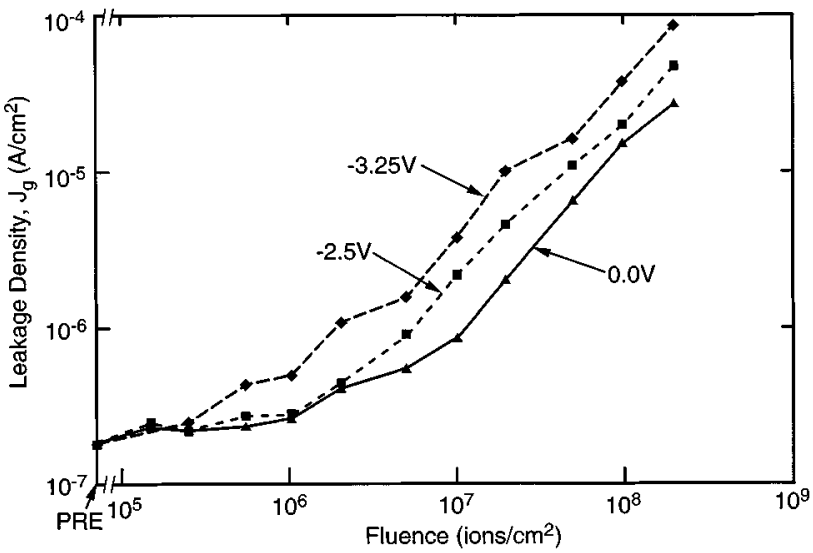

Fig. 3. Plot of $J_{g}$ (extracted at $V_{g}=3.0 \mathrm{~V}$ ) versus fluence for $3.0 \mathrm{~nm}, 10^{-4}$, and $4 \times 10^{-4} \mathrm{~cm}^{2}$ capacitors exposed to I $($ LET $=60)$ with $V_{\text {app }}=-3.25$, -2.5 , or $0.0 \mathrm{~V}$.

ides only exhibit HBD for $V_{\text {app }}$ greater than $\sim 4.8 \mathrm{~V}$ (this could be due to wearout during the irradiation).

Qualitatively, it appears that the electric field required for SEGR/HBD decreases with increasing LET, confirming earlier work in thicker oxides [6]-[8].

Fig. 3 shows heavy-ion $\left({ }^{127} \mathrm{I}\right.$, LET $\left.=60\right)$ induced oxide leakage current density $\left(\mathrm{A} / \mathrm{cm}^{2}\right)$ as a function of fluence and $V_{\text {app }}$. In this figure, $J_{g}$ is an average of at least two capacitors for each $V_{\text {app }}$. Again, results from 3.0-nm oxides with area $=10^{-4} \mathrm{~cm}^{2}$ and $4 \times 10^{-4} \mathrm{~cm}^{2}$ are used. $J_{g}$ is extracted at $V_{g}=-3.0 \mathrm{~V}$. $V_{\text {app }}$ is held at a constant voltage during each subsequent radiation step until a fluence of $2 \times 10^{8}$ ions $/ \mathrm{cm}^{2}$ is achieved. Flux varied from $3 \times 10^{3}$ to $2.4 \times 10^{5}$ ions $/ \mathrm{cm}^{2} / \mathrm{s}$; exposure time varied from 34 to $425 \mathrm{~s}$.

From Fig. 3, it is seen that:

1) there is no critical fluence: increased leakage is observed even at the lowest fluence (a fluence of $1.5 \times 10^{5} / \mathrm{cm}^{2}$ corresponding to roughly 15 and 60 "hits" for the $10^{-4}$ $\mathrm{cm}^{2}$ and $4 \times 10^{-4} \mathrm{~cm}^{2}$ capacitors);

2) leakage density is a strong function of fluence over several decades;

3) there is no critical field: increased leakage is observed even at zero applied voltage;

4) leakage is a function of $V_{\text {app }}$, though it has a weaker dependence than on fluence.

The behavior of the 3.0- and 3.2-nm capacitors was found to be qualitatively the same. A similar dependence of leakage on fluence was reported in thicker oxides by Sexton et al. [6] and at higher fluence in thin oxides by Ceschia et al. [9], [10].

\section{ANALYSIS/DISCUSSION}

Postelectrical stress oxide conduction has recently been modeled by Suñé and Miranda [11], [12] as a localized breakdown that behaves as a quantum point contact (QPC). The current-voltage relationship can be expressed as

$$
I=A \exp ^{-\alpha \phi} \exp ^{\mathrm{BV}}
$$

where $A=4 e / \alpha h, B=\alpha e / 2, e$ is the electronic charge, $h$ is Plank's constant, and $V$ is the voltage drop across the oxide. 


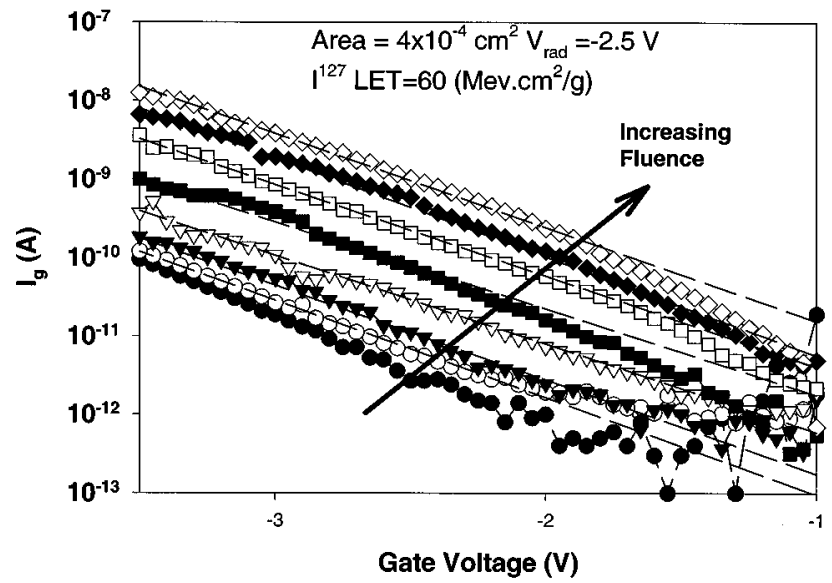

Fig. 4. $I_{g}$ versus $V_{g}$ curves as a function of fluence for a 3.0-nm $4 \times 10^{-4}$ $\mathrm{cm}^{2}$ capacitor exposed to I $(\mathrm{LET}=60)$ with $V_{\mathrm{app}}=-2.5 \mathrm{~V}$. Dashed lines represent fit to theory.

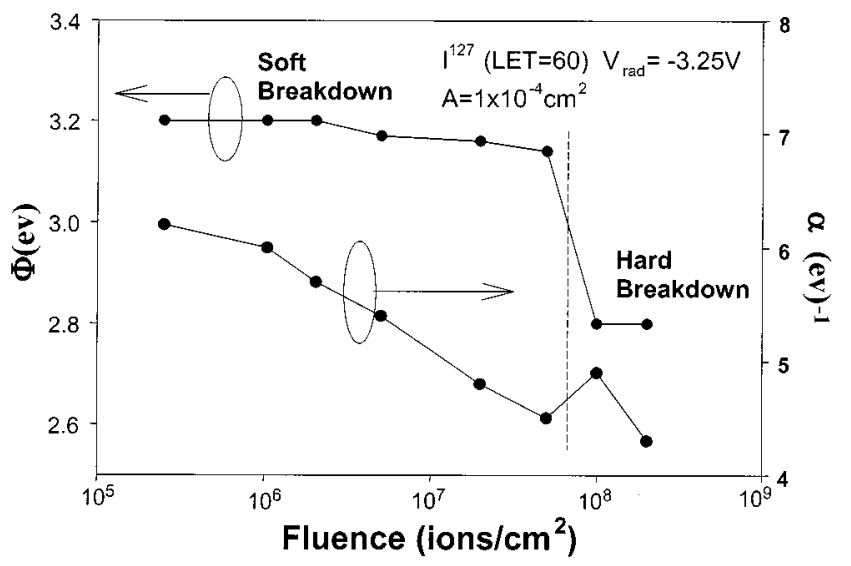

Fig. 5. Extracted $\phi$ (open) and $\alpha$ (solid) versus fluence for a 3.0-nm $10^{-4} \mathrm{~cm}^{2}$ capacitor exposed to I $($ LET $=60)$ with $V_{\text {app }}=-3.25 \mathrm{~V}$.

The two parameters associated with this model are $\phi$, the barrier height of the quantum saddle point contact in units of $\mathrm{eV}$, and $\alpha$, which is correlated to the shape or thickness of the contact and has units of $1 / \mathrm{eV}$. The amount of current that tunnels through an energy barrier is proportional to the inverse of the area of the barrier. The QPC barrier area can be approximated by its thickness times its height. More current flows for thinner (smaller $\alpha$ ) and lower (smaller $\phi$ ) barriers.

Fig. 4 shows a typical example of postirradiation $I_{g}-V_{g}$ curves as a function of fluence for a constant bias during the irradiation. In this case, the $3.0-\mathrm{nm} 4 \times 10^{-4} \mathrm{~cm}^{2}$ capacitors were exposed to ${ }^{127} \mathrm{I}($ LET $=60)$ ions with $V_{\text {app }}=-2.5 \mathrm{~V}$. Simulations using fits to (1) are shown by the dashed lines. Note that the fits are quite good, especially at larger voltages.

Fig. 5 shows the extracted $\phi$ and $\alpha$ as a function of fluence for the same capacitors exposed at $V_{\mathrm{app}}=-3.25 \mathrm{~V}$. $\phi$ and $\alpha$ were extracted by a least squares fit of (1) to postradiation $I-V$ curves such as those shown in Fig. 4: $\alpha$ is related to the slope and $\phi$ is related to $\alpha$ and the $y$-intercept. It is found that $\phi$ is relatively constant as a function of fluence until about $5 \times 10^{7}$ ions $/ \mathrm{cm}^{2}$, where it drops below $3.2 \mathrm{eV}$. According to [11], the drop of $\phi$ below $3.2 \mathrm{eV}$ suggests the onset of hard breakdown. It is also found that $\alpha$ decreases as a function of fluence. A decrease in $\alpha$ can be modeled as the formation of multiple quantum point

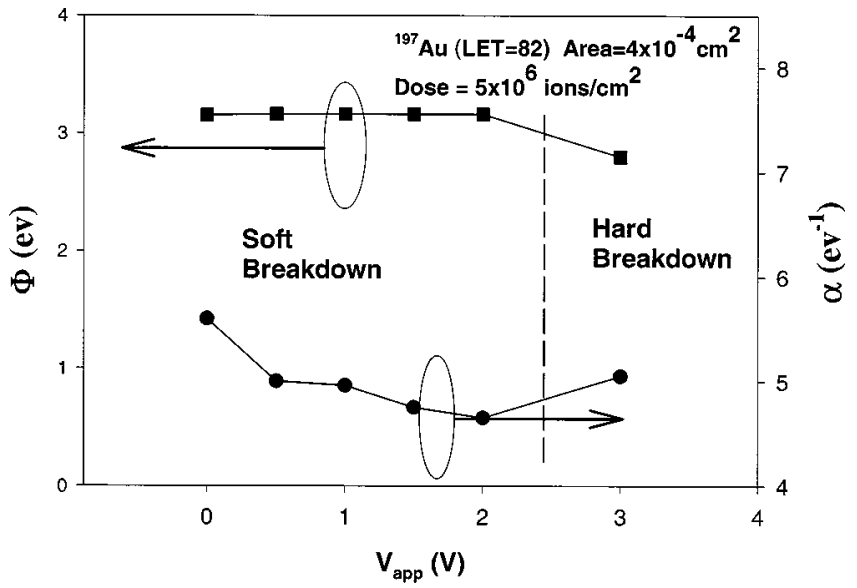

Fig. 6. Extracted $\phi$ (squares) and $\alpha$ (circles) versus $V_{\text {app }}$ for a 3.0-nm $4 \times$ $10^{-4} \mathrm{~cm}^{2}$ capacitor exposed to $\sim 5 \times 10^{6} \mathrm{Au}(\mathrm{LET}=82)$ ions $/ \mathrm{cm}^{2}$.

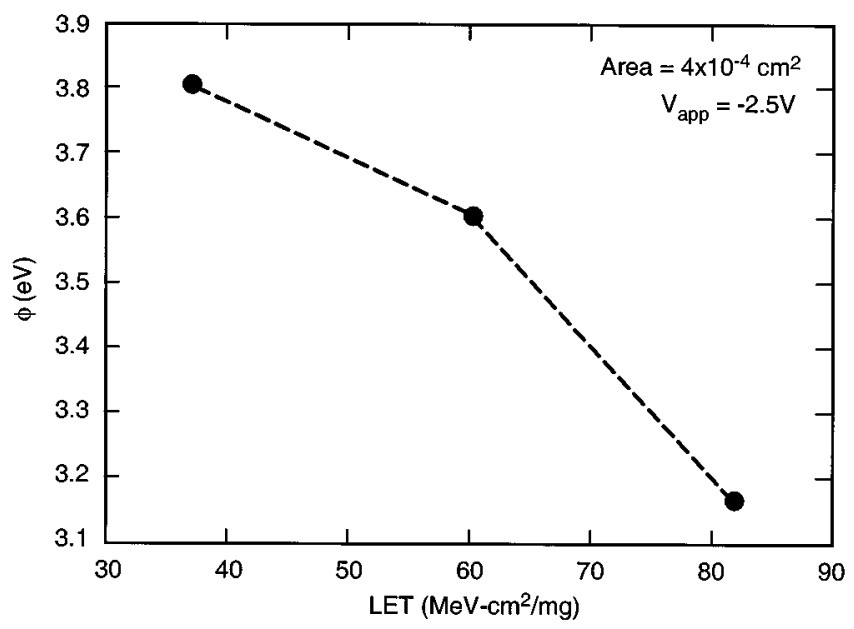

Fig. 7. Extracted $\phi$ versus LET for a 3.0-nm $4 \times 10^{-4} \mathrm{~cm}^{2}$ capacitor exposed to $\sim 5 \times 10^{6}$ ions $/ \mathrm{cm}^{2}$ at $V_{\text {app }}=-2.5 \mathrm{~V}$.

contacts in parallel, each with the same $\phi$. It is reasonable and might even be intuitively expected that for the same LET and voltage, the effect of additional fluence is to create additional similar QPC leakage paths. The total leakage current is a summation of these paths conducting in parallel. This interpretation is consistent with the observed area dependence of the leakage current and with earlier reports [8]-[10].

Fig. 6 shows the extracted values of $\phi$ and $\alpha$ as function of $V_{\text {app }}$, the voltage applied during irradiation. All of the data shown in the figure were obtained at the same ion fluence and LET. Note again that $\phi$ is relatively constant with voltage and that $\alpha$ decreases with voltage. Once again, the drop of $\phi$ below $3.2 \mathrm{eV}$ is accompanied by a large increase in $I_{g}$ (hard breakdown). It has been shown that post-BD leakage is roughly proportional to the power dissipated during $\mathrm{BD}$ and that power dissipation during BD is roughly proportional to $V_{\text {app }}$ [14]. This suggests that the larger $V_{\text {app }}$ produces a more severe damaged region since the power dissipation during the breakdown event is larger. An interpretation of the QPC model suggests that a larger damaged region can be modeled as a QPC with a thinner barrier (smaller $\alpha$ ), thus allowing more current to flow.

Finally, Fig. 7 shows that the $\phi$ is sensitive to LET. This result suggests that higher LET ions produce a more broadly damaged region, perhaps a wider path with a quantum mechanically lower 
barrier height (lower $\phi$ ) that allows more current to flow. Note that the barrier height can be larger than the $\mathrm{Si}-\mathrm{SiO}_{2}$ conduction band offset of $3.2 \mathrm{eV}$. This is a result of quantum confinement that may not necessarily be related to the barrier height of the material in the constricted region [11]. Also, it has recently been reported that voltage drops in the contacts leading to the constricted region may be interpreted as a larger barrier height [12].

From Figs. 1-3, it is found that heavy-ion-induced leakage depends on LET, fluence, and $V_{\text {app }}$, in decreasing importance. In Figs. 4-7, the QPC model is used to model heavy-ion-induced conduction in thin $\mathrm{SiO}_{2}$. It is found that $\alpha$ depends inversely on fluence and $V_{\text {app }}$ and that $\phi$ depends inversely on LET. Our interpretation is that the size of the QPC path is dependent on LET and voltage while the number of paths depends on the fluence. A drop of $\phi$ below $3.2 \mathrm{eV}$ indicates transition from SBD to HBD [11]. As LET increases, this transition appears to occur at lower voltage.

\section{CONCLUSION}

There is controversy in the literature [1], [6]-[9], [16] as to whether heavy-ion irradiation will be a threat to gate oxides in scaled devices. Although the results reported here show that the voltage required for HBD/SEGR is reduced as LET and fluence are increased (confirming earlier reports in thicker oxides [6]-[8]), the field required for the onset of SEGR is outside the range of normal device operation (see also [16]). Future scaling, however, may bring operating voltages into this regime. SEGR may be relevant for applications such as field-programmable gate arrays, which rely on thin dielectrics for programming at high fields. However, oxides may not have to rupture in order for heavy ions to do permanent damage.

What may be more of a concern is RSB. Whether it is actually harmful to the device or circuit will likely depend on the application. There are several situations in which even small amounts of leakage may have an impact on device or circuit operation. It is possible that analog, low power, and memory applications may be susceptible, but it is not clear at what point this would be come a serious issue for digital CMOS. In previous studies of SEGR at low fluence, RSB was noted but was not studied in detail [6]-[8]. (RSB in thin oxides at high fluence has been studied by Ceshia et al. [9], [10].) Sexton et al. [8] referred to RSB as "precursor ion damage" and concluded that, due to the high fluence required, it was not an important failure mechanism. However, the observation of RSB for LET $\geq 60 \mathrm{MeV}-\mathrm{cm}^{2} / \mathrm{mg}$ at relatively low fluence $\left(1.5 \times 10^{5} / \mathrm{cm}^{2}\right)$ and at zero applied fields could be a cause for concern as even "powered down" parts may be susceptible. In addition, because RSB is found to be proportional to area, large total gate areas in future systems may increase susceptibility to RSB.

In the reliability community, electrical-stress-induced soft breakdown is considered the first permanent change in oxide structure [13], [15]. It is defined as destructive because it is not known how the oxide will behave after this first SBD event. Ten-year lifetime extrapolations are based on this first SBD event. Thus, RSB in thin oxides may also properly be considered the end of oxide life.

Although more work is needed to fully understand this damage mechanism and whether it poses a threat to the use of MOS de- vices in space, very recent results show that heavy-ion irradiation can result in a latent reliability problem. At an LET = $60 \mathrm{MeV}-\mathrm{cm}^{2} / \mathrm{mg}\left(737 \mathrm{MeV}{ }^{129} \mathrm{Xe}\right)$ at zero applied field, a fluence of $10^{5}$ ions $/ \mathrm{cm}^{2}$ on $10^{-4} \mathrm{~cm}^{2}$ capacitors ( $\sim 10$ ion "hits") resulted in an over an order of magnitude reduction in timedependent dielectric breakdown lifetime. This effect does not occur for gamma irradiation [17]. So although in the short term, a slight increase in leakage caused by RSB may be tolerated, in the long term, an intrinsically weaker oxide may result.

\section{ACKNOWLEDGMENT}

The authors wish to thank L. Selva, Dr. F. Irom, J. Lehman, T. Minto, and P. Schrock for assistance.

\section{REFERENCES}

[1] A. Paccagnella and M. Ceshia, "Radiation effects in ultra-thin MOS gate oxides," in Proc. IEEE 2000 Materials Reliability and Qualification Workshop, Glendale, CA, Oct. 30-Nov. 1, 2000.

[2] A. Paccagnella, A. Candelori, A. Milani, E. Formigoni, G. Ghidini, F. Pellizzer, D. Drera, P. G. Fuochi, and M. Lavale, "Breakdown properties of irradiated MOS capacitors," IEEE Trans. Nucl. Sci., vol. 43, p. 2609, 1996.

[3] A. Scarpa, A. Paccagnella, F. Montera, G. Ghibaudo, G. Pananakakis, G. Ghidini, and P. G. Fuochi, "Ionizing radiation induced leakage current on ultra-thin gate oxides," IEEE Trans. Nucl. Sci., vol. 44, p. 1818, 1997.

[4] M. Ceschia, A. Paccagnella, A. Cester, A. Scarpa, and G. Ghidini, "Radiation induced leakage current and stress induced leakage current in ultra-thin gate oxides," IEEE Trans. Nucl. Sci., vol. 45, p. 2375, 1998.

[5] L. Larcher, A. Paccagnella, M. Ceschia, and G. Ghidini, "A model of radiation induced leakage current (RILC) in ultra-thin gate oxides," IEEE Trans. Nucl. Sci., vol. 46, p. 1553, 1999.

[6] F. W. Sexton, D. M. Fleetwood, M. R. Shaneyfelt, and P. E. Dodd, "Single event gate rupture in thin oxides," IEEE Trans. Nucl. Sci., vol. 44, p. 2345, 1997.

[7] A. H. Johnston, G. M. Swift, T. Miyahira, and L. D. Edmonds, "Breakdown of gate oxides during irradiation with heavy ions," IEEE Trans. Nucl. Sci., vol. 45, p. 2500, 1998.

[8] F. W. Sexton, D. M. Fleetwood, M. R. Shaneyfelt, P. E. Dodd, G. L. Hash, L. P. Schanwald, R. A. Loemker, M. L. Green, B. E. Weir, and P. J. Silverman, "Precursor ion damage and angular dependence of single event gate rupture in thin oxides," IEEE Trans. Nucl. Sci., vol. 45, p. 2509, 1998.

[9] M. Ceschia, A. Paccagnella, S. Sandrin, G. Ghidini, J. Wyss, M. Lavale, and O. Flament, "Low field leakage current and soft breakdown in ultra thin gate oxides after heavy ions, electrons, or x-ray irradiation," IEEE Trans. Nucl. Sci., vol. 47, p. 566, 2000.

[10] M. Ceschia, A. Paccagnella, M. Turrini, A. Candelori, G. Ghidini, and J. Wyss, "Heavy ion irradiation of thin gate oxides," IEEE Trans. Nucl. Sci., vol. 47, p. 2648, 2000, submitted for publication.

[11] J. Sune and E. Miranda, "Post soft breakdown conduction in $\mathrm{SiO}_{2}$ gate oxides," in Int. Electron Devices Meeting (IEDM) 2000 Tech. Dig., pp. $553-556$.

[12] E. Miranda and J. Sune, "Analytic modeling of leakage current through multiple breakdown paths in $\mathrm{SiO}_{2}$ films," in Proc. 39th Annual IRPS, 2001, pp. 367-379.

[13] E. Y. Wu, W. W. Abadeer, L.-K. Han, S. H. Lo, and G. Hueckel, "Challenges for accurate reliability projections in the ultra-thin oxide regime," in Proc. 37th Annual IRPS, 1999, p. 57.

[14] M. A. Alam, B. Weir, J. Bude, P. Silverman, and D. Monroe, "Explanation of soft and hard breakdown and its consequences for area scaling," in Int. Electron Devices Meeting (IEDM) 1999 Tech. Dig., pp. 449-452.

[15] B. E. Weir et al., "Gate oxides in 50nm devices: Thickness uniformity improves projected reliability," in Int. Electron Devices Meeting (IEDM) 1999 Tech. Dig., pp. 437-440.

[16] L. W. Massengill et al., "Heavy-ion-induced breakdown in ultra-thin gate oxides and high-k dielectrics," IEEE Trans. Nucl. Sci., vol. 48, Dec. 2001 .

[17] J. S. Suehle, B. Wang, J. F. Conley, Jr, E. M. Vogel, P. Roitman, C. E. Weintraub, A. H. Johnston, and J. B. Bernstein, "Latent reliability degradation observed in ultra-thin oxides after heavy-ion irradiation,", submitted for publication. 